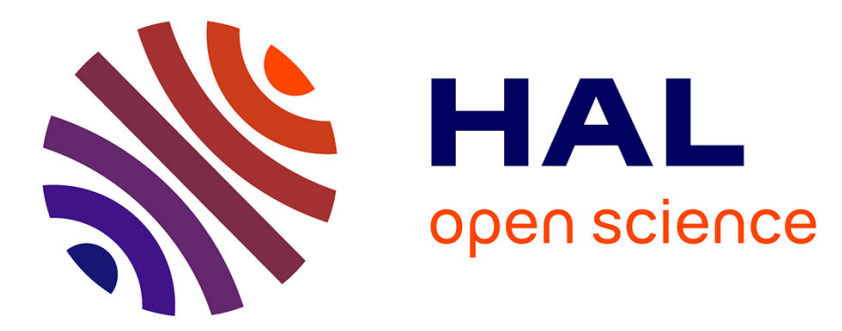

\title{
A Temporal-Causal Network Model for the Internal Processes of a Person with a Borderline Personality Disorder
}

\author{
Maria Hoțoiu, Federico Tavella, Jan Treur
}

\section{To cite this version:}

Maria Hoțoiu, Federico Tavella, Jan Treur. A Temporal-Causal Network Model for the Internal Processes of a Person with a Borderline Personality Disorder. 14th IFIP International Conference on Artificial Intelligence Applications and Innovations (AIAI), May 2018, Rhodes, Greece. pp.362-373, 10.1007/978-3-319-92007-8_31. hal-01821027

\section{HAL Id: hal-01821027 \\ https://hal.inria.fr/hal-01821027}

Submitted on 22 Jun 2018

HAL is a multi-disciplinary open access archive for the deposit and dissemination of scientific research documents, whether they are published or not. The documents may come from teaching and research institutions in France or abroad, or from public or private research centers.
L'archive ouverte pluridisciplinaire HAL, est destinée au dépôt et à la diffusion de documents scientifiques de niveau recherche, publiés ou non, émanant des établissements d'enseignement et de recherche français ou étrangers, des laboratoires publics ou privés. 


\title{
A Temporal-Causal Network Model for the Internal Processes of a Person with a Borderline Personality Disorder
}

\author{
Maria Hoțoiu ${ }^{1}$, Federico Tavella ${ }^{1,2}$, and Jan Treur ${ }^{1}$ \\ ${ }^{1}$ Behavioural Informatics Group, Vrije Universieit Amsterdam \\ ${ }^{2}$ Department of Mathematics, University of Padua \\ mariahotoiu@gmail.com federicotavella.7@gmail.com \\ $j \cdot$ treur@vu.nl
}

\begin{abstract}
This paper presents a computational network model for a person with a Borderline Personality Disorder. It was designed according to a Network-Oriented Modeling approach as a temporal-causal network based on neuropsychological background knowledge. Example simulations are discussed. The model was verified based on Mathematical Analysis of stationary points.
\end{abstract}

Keywords: Borderline personality disorder, temporal-causal network model.

\section{Introduction}

Borderline Personality Disorder (often referred as BPD) is a psychiatric disorder characterized by a continuous pattern of swinging moods, self-image and behavior (NIH website, 2017). People suffering from this disorder may also experience acute episodes of anger, depression and anxiety which can have different duration (typically from a few hours to days). These symptoms often lead to impulsive actions and problems in social interactions (Lis et al., 2013; Mellisa et al., 2017).

As for a relevant amount of mental disorders, people with borderline personality disorder may experience different symptoms based on disorder acuity and severity. In the case of BPD, two of the most common indicators are mood swings and display uncertainty about how they see themselves and their role in the world (NIH website, 2017). People with borderline personality disorder also tend to view (i.e., interpret) things in a "dichromatic" way, such as all black or white. Consequently, their idea of other people can also change very quickly: an individual who is seen as a friend one day may be considered an enemy or traitor the day after. These fluctuating emotional states can lead to intense and unstable relationships. Other symptoms may include:

- A pattern of intense and unstable relationships with relatives, friends, and loved ones, often swinging from extreme closeness and love (idealization) to extreme dislike or anger (devaluation) 
- Feelings of dissociation (Brand et al., 2014), such as feeling cut off from oneself, seeing oneself from outside one's body, or feelings of unreality

- Self-harming behavior and recurring thoughts of suicidal behaviors or threats;

- Difficulty imagining embodied others, i.e. different empathy levels (Haas et al, 2015; Dammann et al., 2011)

- Inappropriate/intense anger or problems controlling anger

- Distorted and unstable self-image or sense of self (Dammann et al., 2011)

As previously mentioned, not all the subjects with borderline personality disorder experience every symptom. Some individuals experience only a few symptoms, while others have many. These symptoms can be triggered by everyday life events. Moreover, studies show that people with borderline personality disorder can have structural and functional changes in the brain (Soloff et al., 2017) specially in the areas that control impulses and emotional regulation (NIH website, 2008). However, it is not clear whether these changes are risk factors for the disorder, or caused by the disorder.

This paper presents a computational network model for a person with borderline. It was designed as a temporal-causal network based on the Network-Oriented Modeling approach described in (Treur, 2017). First in Section 2 some neuropsychological background is described. In Section 3 the model is introduced. Section 4 discusses some example simulations. In Section 5 it is described how the model was verified based on Mathematical Analysis.

\section{Neuropsychological Background}

The findings of numerous studies and research papers from Psychiatry, Cognitive and Social Neuroscience have been used to obtain a justifiable basis for the design of the computational model. The primary criteria for assessing the diagnosis of BPD according to (Ellison et al., 2016; American Psychiatric Association, 2013) are (1) behavioral dysregulation, including impulsivity, excessive and inappropriate anger, self-harming and suicidality, (2) a history of failed relationships and feelings of "emptiness", (3) affective dysregulation including excessive mood lability, paranoia and fear of abandonment. One explanation offered by (De Meulemeester et al., 2016) for the interpersonal problems that these people experience is identity diffusion, fundamentally characterized by problems with self-other boundaries. According to the study, patients suffering from BPD displayed an instability in their sense of self and identity.

A factor contributing to the affective instability of BPD, according to a study by (Koenigsberg et al., 2010), is that, compared to healthy controls, patients with BPD do not engage the cognitive control regions when employing a distancing strategy to regulate emotional reactions. Anxiety and mistrust, as well as fear of other people's intentions are also common findings in the studies regarding BPD. One such study by (KingCasas et al., 2008) involved a mix sample of BPD patients and healthy controls playing a multi round economic exchange game. The findings suggest that BPD subjects express significantly lower levels of self-reported trust relative to healthy controls, as well as negative expectations of social partners, which was also implied by their decisions throughout the game. When studying the link between empathy and social attributions 
in BPD patients, (Homan et al., 2017) found that BPD patients display the tendency to attribute behavior to traits rather than context, meaning that they have a reduced empathic capacity.

The implications of these studies to the computational model are further discussed is Section 3 and Section 4.

\section{The Temporal-Causal Network Model}

In order to conceptualize the internal processes, as well as the interaction with an external person, of a person suffering from BPD, a temporal-causal network modelling approach was used, as described in (Treur, 2016). Causal modelling, causal reasoning and causal simulation have a long tradition in AI; e.g., (Kuipers and Kassirer, 1983; Kuipers, 1984; Pearl, 2000). The Network-Oriented Modelling approach based on temporal-causal networks described in (Treur, 2016) on the one hand can be viewed as part of this causal modelling tradition, and on the other hand in the perspective of mental states and their causal relations as described in Philosophy of Mind; e.g., (Kim, 1996). It is a widely usable generic AI modelling approach that distinguishes itself by incorporating a dynamic and adaptive temporal perspective, both on states and on causal relations. This dynamical perspective enables modelling of cyclic and adaptive networks, and also of timing of causal effects. This enables modelling by adaptive causal networks for connected mental states and for social interaction. Temporal-causal network models can be represented at two levels: conceptual and numerical. These is discussed subsequently in Sections 3.1 and 3.2.

The following mechanisms based on different theories from the literature are incorporated in the model:

- mirror neuron systems (Iacoboni and Dapretto 2006; Iacoboni, 2008)

- control neurons with self-other distinction and control function (Iacoboni, 2008; Brass and Spengler, 2009)

- emotion integration (Grèzes and de Gelder, 2009; Grèzes et al., 2009)

- regulation of enhanced sensory processing sensitivity, in particular for face expressions (Neumann et al., 2006), (Spezio et al., 2007; Baker et al., 2008; Corden et al., 2008)

- empathic responding using mirror neurons, self-other distinction and emotion integration (De Vignemont and Singer, 2006; Singer and Leiberg, 2009)

In order to have an adequate social interaction, all these mechanisms should function properly. Since they are correlated to one another, the malfunction of any one of them can lead to problems in the social functioning of the individual. People who suffer from BPD display faults in some of these mechanisms, as described in Section 2. They do not express the tendency to avoid stimuli, even if they are unpleasant, therefore no avoiding mechanism was included in the model. The manner in which these faults are translated into the model is described in Section 4. 


\subsection{The Conceptual Representation of the Network Model}

Temporal-causal networks can be represented in a conceptual manner declaratively in either a graphical form or in a matrix form. The elements of a conceptual representation of a design are:

- the states $Y$ of the network

- the connections between these states

- connection weights $\omega_{X, Y}$ which characterize the different strengths of these connections

- a speed factor $\eta_{Y}$ for each state, expressing how fast this state can change

- for each state $Y$ a combination function $\mathbf{c}_{Y}(. .$.$) indicating how the multiple im-$ pacts from the states with outgoing connections to $Y$ combine into a single impact on that state.

In the graphical conceptual representation, states are displayed as nodes and connections as arrows, the pointed end of the arrow indicating the direction of the causal impact. The graphical conceptual representation depicted in Fig. 1 describes the states and their connections in a person suffering from BPD. The above mentioned concepts connection weights, speed factors and combination functions are labels for the arrows (connection weights) and nodes (speed factors and combination functions) of this graph, so that a fully specified graphical conceptual representation gets the form of a labeled graph. There are six types of states in the presented model:

- world states ws, indicating an external stimulus, in this case $\mathrm{ws}_{s}$ for the stimulus $s$ and $\mathrm{ws}_{B}$ for a person $B$

- sensor states $\operatorname{ss}_{X}$ (with $X=s$ or $X=B$ ) for the sensing of these two external stimuli

- sensory representation states $\operatorname{srs}_{X}$ (with $X=s$ or $X=B$ ) formed based on the sensor states, but also within the person itself and body states of aggression and anxiety

- preparation states $\mathrm{ps}_{X}$ for expressing the body states of anxiety $(X=a n x)$ and aggression $(X=a g g)$, as well as for communicating to person $B(X=B)$

- control states $\operatorname{cs}_{X}$, which regulate the actual expression of anxiety and aggression $(X=$ self $)$ as well the self-other distinction between the person and the other person $(X=B, s)$

- execution or expression states es ${ }_{X}$ for anxiety and aggression and execution state $\operatorname{esc}_{X}$ for communication to person $B$

Besides the connections described previously, there are also two loops: the as-if body loops between the preparation states for expressing anxiety and aggression and their sensory representation states, which adapt the internal body map, as described in (Treur, 2016). 


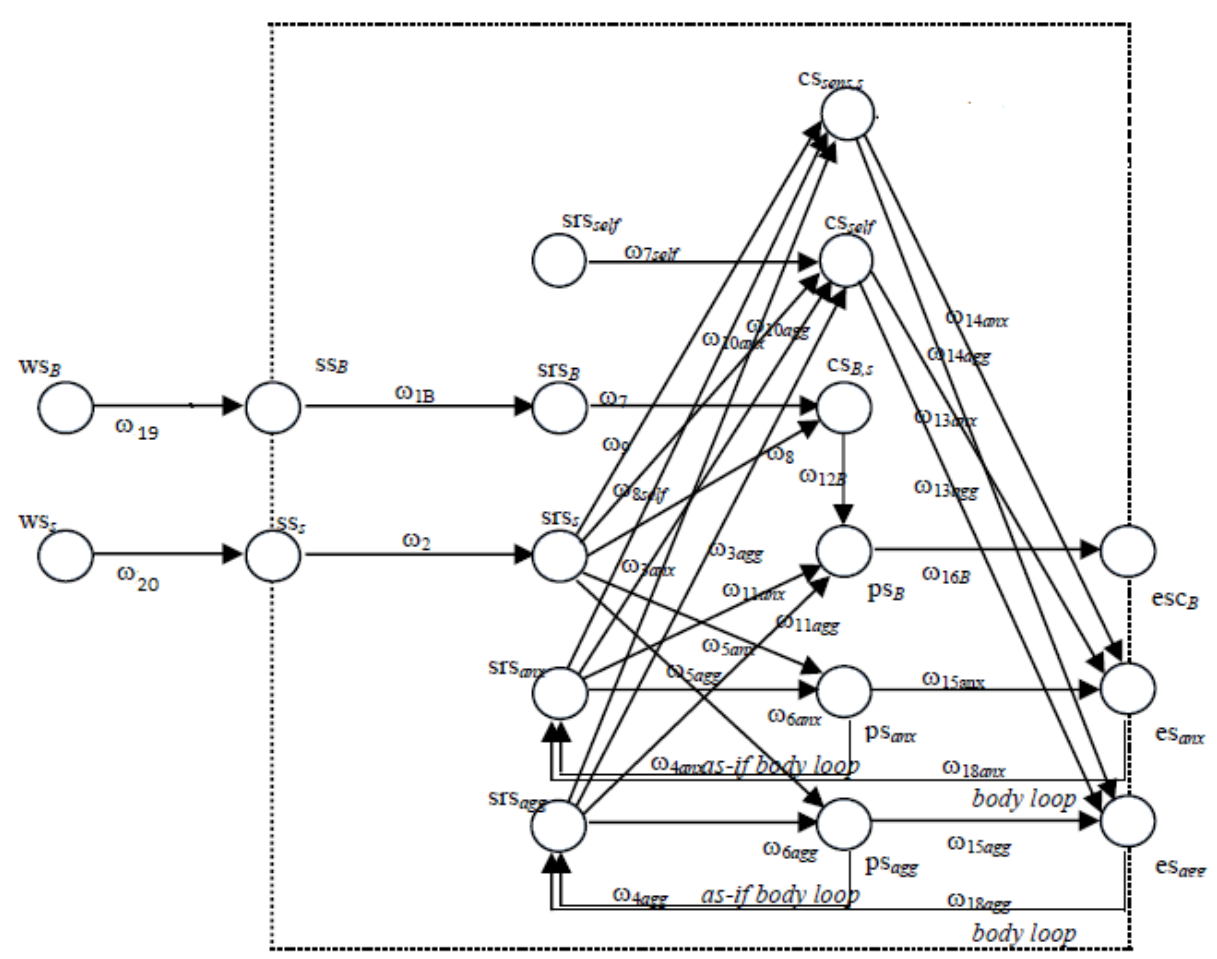

Fig. 1. The graphical conceptual representation of the temporal-causal network model

\subsection{Numerical Representation of the Network Model}

In order to obtain a basis for simulation and further mathematical analysis of the model, the conceptual representation can be transformed into a numerical representation in a systematic manner, as described in (Treur, 2016), as follows:

- The value of each state $Y$ at each time point $t$ is denoted by $Y(t)$

- The impact from any connection from a state $X_{i}$ to a state $Y$ at time point $t$ is defined as

$$
\operatorname{impact}_{X i, Y}(t)=\omega_{X_{i}, Y} X_{i}(t)
$$

where $\omega_{X_{i}, Y}$ is the weight of the connection from $X_{i}$ to $Y$

- The total impact of all the connections $X_{1}, \ldots, X_{k}$ to a state $Y$ at time $t$ is

$$
\operatorname{aggimpact}_{Y}(t)=\mathbf{c}_{Y}\left(\operatorname{impact}_{X_{1}, Y}(t), \ldots, \operatorname{impact}_{X_{\mathrm{k}}, Y}(t)\right)
$$

where $\mathbf{c}_{Y}(\ldots)$ is the combination function

- The change of the state $Y$ from the time step $t$ to the next time point $t+\Delta t$ is represented by the following difference equation:

$$
Y(t+\Delta t)=Y(t)+\eta_{Y}\left[\operatorname{aggimpact}_{Y}(t)-Y(t)\right] \Delta t
$$


where $\eta_{Y}$ is the speed factor of the state $Y$, or by the following differential equation:

$$
\mathbf{d} Y(t) / \mathbf{d} t=\eta_{\mathrm{Y}}\left[\operatorname{aggimpact}_{Y}(t)-Y(t)\right]
$$

As an example, by following the pattern described above based on formulae (1), (2), (3) and (4) the difference and the differential equation for $\mathrm{ps}_{\mathrm{B}}$ are:

$$
\begin{gathered}
\operatorname{ps}_{B}(t+\Delta t)=\operatorname{ps}_{B}(t)+\eta_{\mathrm{ps}_{B}}\left[\mathrm{c}_{\mathrm{ps} B}\left(\omega_{11 \text { anx }} \operatorname{srs}_{a n x}(t), \omega_{11 \text { agg }} \operatorname{srs}_{a g g}(t), \omega_{12 B} \operatorname{cs}_{B, s}(t)\right)-\mathrm{ps}_{\mathrm{B}}(\mathrm{t})\right] \Delta t(5) \\
\operatorname{dps}_{\mathrm{B}}(\mathrm{t}) / \mathbf{d t}=\eta_{\mathrm{ps} B}\left[\mathrm{c}_{\mathrm{ps} B}\left(\omega_{11 \text { anx }} \operatorname{srs}_{\text {anx }}(t), \omega_{11 \text { agg }} \operatorname{srs}_{a g g}(t), \omega_{12 B} \operatorname{cs}_{B, s}(t)\right)-\operatorname{ps}_{\mathrm{B}}(\mathrm{t})\right]
\end{gathered}
$$

Each state of the model, except for the world states which have no incoming connections, gets a difference and a differential equation assigned. The interaction between these equations describe the behavior of the model. For the model considered here there are 16 coupled difference and differential equations.

The combination function used for all the states with only one incoming impact is the identity function id(...) as described in (Treur, 2016, Ch 2): $\mathbf{c r}(V)=\mathbf{i d}(V)=V$. By using this combination function, based on (1) to (4) the difference and the differential equation for $\mathrm{srs}_{\mathrm{B}}$, for example, is the following:

$$
\begin{aligned}
& \operatorname{srs}_{\mathrm{B}}(t+\Delta t)=\operatorname{srs}_{\mathrm{B}}(t)+\eta_{\operatorname{srs}_{B}}\left[\omega_{1 B} \operatorname{ss}_{\mathrm{B}}(t)-\operatorname{srs}_{\mathrm{B}}(t)\right] \Delta t \\
& \mathbf{d s r s} \mathrm{B}_{\mathrm{B}}(t) / \mathbf{d} t=\eta_{\operatorname{srs}_{B}}\left[\omega_{1 B} \operatorname{ss}_{\mathrm{B}}(t)-\operatorname{srs}_{\mathrm{B}}(t)\right]
\end{aligned}
$$

For the states which have multiple incoming impacts the combination function used was the advanced logistic sum combination function alogistic(...), described in (Treur, 2016) as follows:

$$
\operatorname{cy}_{\mathbf{Y}}\left(V_{1}, \ldots, V_{k}\right)=\operatorname{alogistic}_{\sigma, \tau}\left(V_{1}, \ldots V_{k}\right)=\left[\left(1 /\left(1+\mathrm{e}^{-\sigma\left(V_{1}, \ldots V_{k}-\tau\right)}\right)\right)-1 /\left(1+\mathrm{e}^{\sigma \tau}\right)\right]\left(1+\mathrm{e}^{-\sigma \tau}\right)
$$

The parameters $\tau$ and $\sigma$ of this advanced logistic function represent the threshold and the steepness. This function has the property that it maps 0 values to 0 and it also keeps the values between 0 and 1 . With this combination function (7), based on (1) to (4) the following difference and differential equations for $\mathrm{ps}_{\mathrm{B}}$, for example, are obtained:

$\operatorname{ps}_{B}(t+\Delta t)=\operatorname{ps}_{B}(t)+$

$\eta_{\mathrm{ps}_{B}}\left[\operatorname{alogistic}_{\sigma, \tau}\left(\omega_{11 a n x} \operatorname{srs}_{a n x}(t), \omega_{11 \text { agg }} \operatorname{srs}_{a g g}(t), \omega_{12 B} \operatorname{cs}_{B, s}(t)\right)-\operatorname{ps}_{\mathrm{B}}(\mathrm{t})\right] \Delta t$

$\mathbf{d p s}_{\mathrm{B}}(\mathrm{t}) / \mathbf{d t}=\eta_{\mathrm{ps}_{B}}\left[\operatorname{alogistic}_{\sigma, \tau}\left(\omega_{11 \text { anx }} \operatorname{srs}_{a n x}(t), \omega_{1 \text { lagg }} \operatorname{srs}_{a g g}(t), \omega_{12 B} \operatorname{cs}_{B, s}(t)\right)-\operatorname{ps}_{\mathrm{B}}(\mathrm{t})\right]$

\section{Simulation Results}

The numerical representation presented above was implemented in Matlab, in order to obtain a realistic picture of how the mechanisms discussed in Section 3 interact in the case of a person suffering from BPD. The traits presented in Section 2 are translated into the model by constraints of some of the parameters, as shown in Table 1, thus providing the data at the basis of the simulation. 
Following the reasoning presented in (Treur, 2016, Ch 10) a reduced self-other distinction means that the connections between $\operatorname{srs}_{s}$ and $\operatorname{cs}_{B, s}$ and $\operatorname{srs}_{B}$ and $\operatorname{cs}_{B, s}$ are weak, therefore the values of $\omega_{7}, \omega_{8}$ are low. The intensity of the anger and of the anxiety experienced by these people implicates that the connections between their corresponding sensory representations srs ${ }_{a n x}$ and $\operatorname{srs}_{a g g}$ and the control state for sensing $\mathrm{cs}_{\text {sens,s }}$ are weak, as well as the connections between the control state for monitoring $\mathrm{cs}_{\text {self }}$ and $\mathrm{srs}_{\text {anx }}$ and $\operatorname{srs}_{\text {agg }}$ with $\omega_{10 a n x}, \omega_{10 a g g}, \omega_{3 a g g}, \omega_{3 a n x}$ are low. The connection between the sensory representation $\operatorname{srs}_{s}$ of a stimulus $s$ and the control state for sensing $\mathrm{cs}_{\text {sens,s }}$ is also weak, with $\omega_{9}$ low, since people suffering from BPD do not exhibit a distancing behavior.

The poor monitoring of emotions translates into weak connections between the sensory representations of the agent itself $\operatorname{srs}_{\text {self }}$, as well as the sensory representation $\operatorname{srs}_{s}$ of stimulus $s$, and the control state for the agent itself $\operatorname{cs}_{\text {self }}$ with $\omega_{7 \text { self }}$ and $\omega_{8 \text { self }}$ low. Reduced self-control implicates that the connections coming from the control state $\mathrm{cs}_{\text {sens, }, s}$, as well as from $\mathrm{cs}_{\text {self }}$, to the expression states es $\mathrm{en}_{\text {anx }}$ and $\mathrm{es}_{\text {agg }}$ are weak, so $\omega_{13 a n x}$, $\omega_{13 a g g}, \omega_{14 a n x}$, and $\omega_{14 a g g}$ are low. All the weights of the connections except for those in Table 1 are 1.

Table 1. Connection weights and their values

\begin{tabular}{|c|c|c|c|}
\hline Connection & Value & Connection & Value \\
\hline$\omega_{7}$ & 0.5 & $\omega_{13 \text { anx }}, \omega_{13 \text { agg }}$ & -0.2 \\
\hline$\omega_{8}$ & 0.5 & $\omega_{\text {self }, \omega 8 \text { self }}$ & 0.2 \\
\hline$\omega_{3 a g g}, \omega_{3 a n x}$ & 0.2 & $\omega_{14 a n x}, \omega_{14 a g g}$ & -0.2 \\
\hline$\omega_{10 a n x}, \omega_{10 a g g}$ & 0.2 & $\omega_{9}$ & 0.2 \\
\hline
\end{tabular}

In order to incorporate the impulsivity trait in the implementation, the speed factors of the control states $\mathrm{cs}_{s e n s, s}, \mathrm{cs}_{\text {self }}$ and $\mathrm{cs}_{B, s}$ are adjusted as being lower than for the other states, for which it is 1 . The speed factor of the states regarding communication, $\mathrm{ps}_{B}$ and $\operatorname{esc}_{B}$ are also a little lower than one, since verbalizing is a little difficult for people who suffer from BPD so it is most likely to happen after the body expressions of anxiety and aggression. The parameters for the advanced logistic sum combination function corresponding to each state are shown in Table 2.

Table 2: Parameters for the combination function alogistic $_{\sigma, \tau}(.$.$) for each state$

\begin{tabular}{|c|c|c|c|c|c|}
\hline state & $\sigma$ & $\tau$ & state & $\sigma$ & $\tau$ \\
\hline $\mathrm{CS}_{\text {sens,s }}$ & 1 & 0.2 & SrSanx & 5 & 0.4 \\
\hline pSB & 4 & 0.2 & pSanx & 5 & 0.4 \\
\hline $\mathrm{csB}, \mathrm{s}$ & 2 & 0.2 & esanx & 5 & 0.4 \\
\hline srsagg & 5 & 0.4 & $\mathrm{ps}_{\mathrm{agg}}$ & 5 & 0.4 \\
\hline es agg & 5 & 0.4 & $\mathrm{cS}_{\text {self }}$ & 1 & 0.2 \\
\hline
\end{tabular}

The step size is set at $\Delta t=0.5$. The initial values of the states are 0 , except for the input states $\mathrm{wS}_{\mathrm{B}}$ and $\mathrm{ws}_{s}$ which have either the value of 0.2 or 0 , equivalent to no stimulus. The value of 0.2 is chosen so low in order to illustrate the strong impact a weak stimulus has on a person suffering from BPD. The results of the simulation for the values discussed above are shown in Fig. 2. 
In the first part of the simulation, all the values go up, first the values of the stimuli related states, $\mathrm{ss}_{B}$ and $\mathrm{ss}_{s}$ which are equal, so their graphical representations are overlapping, making only $\mathrm{ss}_{B}$ visible. The same is happening with $\operatorname{srs}_{B}$ and $\operatorname{srs}_{s}$. Even though the value of the input is as low as 0.2 , the values of the states for preparation $\mathrm{ps}_{\text {anx }}$ and $\mathrm{ps}_{a g g}$ and expression es $\mathrm{s}_{a n x}$ and $\mathrm{es}_{\text {agg }}$ climb really fast to values as high as 0.8 and 0.9 . This illustrates the power of a small stimuli, when self-control is not working correctly. Note that, since aggression and anxiety are expected to play an equal role in this model, their graphical representations are overlapping. The control states for emotion regulation and suppression and self-other distinction $\mathrm{cs}_{\text {sens, },}, \mathrm{cs}_{B, s}$ and $\mathrm{cs}_{\text {self }}$ are always low, as expected. The preparation $\mathrm{ps}_{B}$ communication to person $B \operatorname{esc}_{B}$ happen after the expressions of anxiety and aggression, corresponding to the behavior previously described.

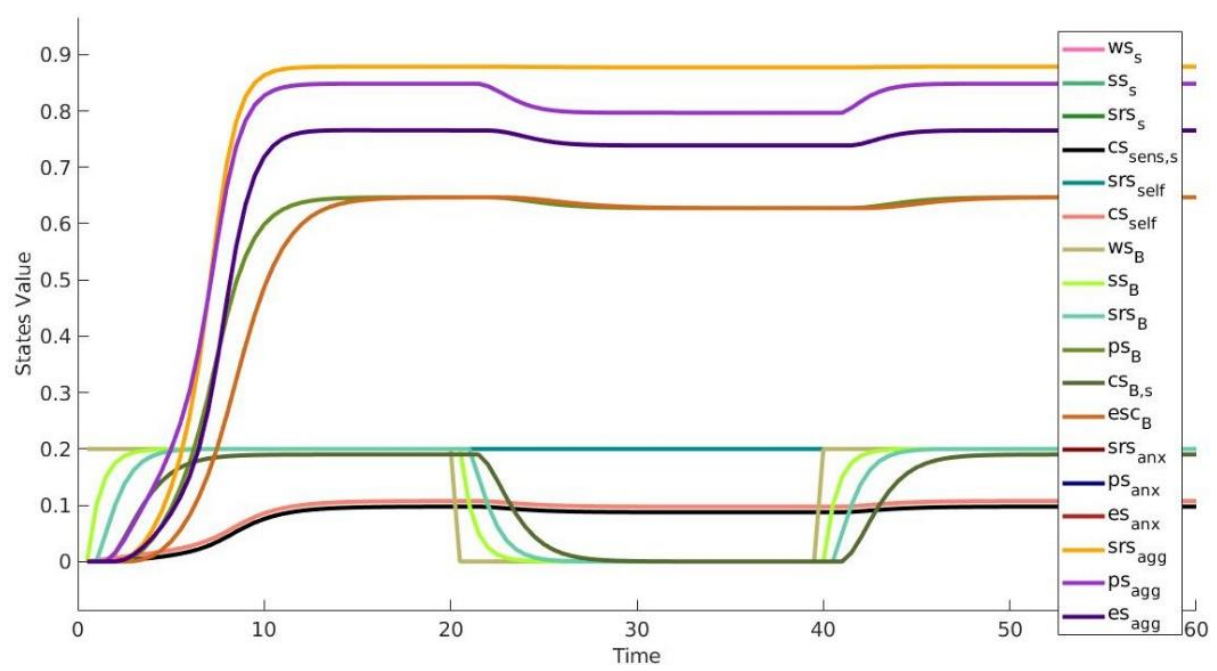

Fig. 2. Results of the simulation

After the first 10 time steps, the stimuli go away, meaning that the values of $\mathrm{ws}_{s}$ and $\mathrm{ws}_{B}$ become 0 . In a normal person, this would imply that the values of all the other states should decrease to 0 , but here the states related to anger and anxiety drop only very little , as expected. The sensory representation states for anxiety $\operatorname{srs}_{a n x}$ and aggression $\operatorname{srs}_{a g g}$ are always high in value, illustrating the constantly disturbed inner state people suffering from BPD experience. Since the value of the sensory representation state is high and the values of the control states are low, the expression of the feelings is also high, even if there are no stimuli present. This process of stimuli/no stimuli is repeated throughout the simulation a few times, with the same results. 


\section{$5 \quad$ Verification by Mathematical Analysis}

In order to verify the implementation of the model, a mathematical analysis of the equilibria was performed. A model is in equilibrium at a time point $t$ if all the states of the model have a stationary point at that time $\mathrm{t}$. A state $\mathrm{Y}$ has a stationary point at a time $\mathrm{t}$ if $Y(t+\Delta t)=Y(t)$, for a small $\Delta t$. By considering the differential equation representation for a temporal-causal network model (see (1) to (4) in Section 3.2), a state $Y$ has a stationary point a time $t$ if and only:

$$
Y(t)=\mathbf{c}_{Y}\left(\omega_{X_{1}, Y} X_{1}(t), \ldots, \omega_{X_{k}, Y} X_{k}(t)\right)
$$

where $\mathbf{c}_{Y}()$ is the combination function for the state $Y$ and $X_{1}, \ldots, X_{k}$ are the states which have an impact on $Y$; see (Treur, 2016, Ch 12). Following this representation if we consider the equilibrium equations for all the states $X_{i}$ of the model, by leaving out the $t$ and denoting the values as constants $\underline{\mathbf{X}}_{i}$, we get from (9) for all states $Y$ that an equilibrium is a solution $\left(\underline{\mathbf{X}}_{1}, \ldots, \underline{\mathbf{X}}_{n}\right)$ of the following $n$ equations:

$$
\begin{aligned}
& \underline{\mathbf{X}}_{1}=\mathbf{c}_{X_{1}}\left(\omega_{X_{1}, X_{1}} \underline{\mathbf{X}}_{1}, \ldots, \omega_{X_{n}, X_{1}} \underline{\mathbf{X}}_{n}\right) \\
& \underline{\mathbf{X}}_{\mathrm{n}}=\mathbf{c}_{X_{n}}\left(\omega_{X_{1}, X_{n}} \underline{\mathbf{X}}_{1}, \ldots, \omega_{X_{n}, X_{n}} \underline{\mathbf{X}}_{n}\right)
\end{aligned}
$$

The model discussed here has 18 states, out of which two are world states, therefore 16 equilibrium equations. For example, the equilibrium equation for the state $\mathrm{ss}_{B}$, which has the identity combination function, is:

$$
\underline{\mathbf{S S}}_{B}=\boldsymbol{\omega}_{19} \underline{\mathbf{W S}}_{B}
$$

For the other states which use the identity combination function, the equilibrium equations are similar. For the states which use the combination function alogistic $\sigma_{\sigma, \tau}(.$.$) (see$ (7) in Section 3.2), the equilibrium equations are similar to this one, which is for the state $\mathrm{ps}_{B}$ :

$$
\mathbf{p S}_{B}=\operatorname{alogistic}_{\sigma, \tau}\left(\omega_{11 \text { anx }} \underline{\mathbf{S r S}}_{a n x}, \omega_{11 \text { agg }} \underline{\mathbf{S r S}}_{a g g}, \omega_{12 B} \underline{\mathbf{c S}}_{B, s}\right)
$$

Due to the 10 equations that include a logistic function, the equilibrium equations cannot be solved analytically in an explicit manner, but they still can be used for verification of the model. Since the model discussed here has an alternating presence of the stimuli, the model reaches stationary points several times.

In order to be able to analyse the stationary points of the model, the number of time steps for observation was increased from 120 to 1200 , so that the values of the states would be observed more often. The stimulus is removed and added again once every 200 time steps, so we would expect that the model reaches stationary points 6 times in the simulation. This type of pattern is called a limit cycle, with state values changing all the time. In a limit cycle, each state fluctuates between a minimum and a maximum value. When it reaches the time points for either a minimum or a maximum, each state should have a stationary point, which means that the equation for a stationary point can be verified. The stationary point equations were fulfilled for all the states of the model, 
with a very high accuracy, as can be seen in Table 3 which is evidence that the implemented model does what is expected.

Table 3. Overview of the outcomes of the verification of the stationary points

\begin{tabular}{|r|c|c|c|c|c|c|c|}
\hline \multicolumn{1}{|c|}{ State } & $\mathbf{S S}_{B}$ & $\mathbf{s r s}_{B}$ & $\mathbf{S s}_{s}$ & $\mathbf{s r s}_{s}$ & $\mathbf{s r s}_{\text {anx }}$ & $\mathbf{s r s}_{a g g}$ & cs $_{\text {self }}$ \\
\hline Maxima & & & & & & & \\
\hline Time point & 196 & 196 & 196 & 196 & 196 & 196 & 196 \\
\hline Value & 0.2 & 0.2 & 0.2 & 0.2 & 0.878487 & 0.878487 & 0.107426 \\
\hline Aggimpact & 0.2 & 0.2 & 0.2 & 0.2 & 0.878487 & 0.878487 & 0.107426 \\
\hline Deviation & 0 & 0 & 0 & 0 & 0 & 0 & 0 \\
\hline Minima & & & & & & & 406 \\
\hline Time point & 406 & 406 & 406 & 406 & 406 & 406 & 0.087503 \\
\hline Value & $9.96 \mathrm{e}-61$ & $1.97 \mathrm{e}-58$ & $9.96 \mathrm{e}-61$ & $1.97 \mathrm{e}-58$ & 0.877405 & 0.877405 & 0.087503 \\
\hline Aggimpact & 0 & 0 & 0 & 0 & 0.877405 & 0.877405 & 0.08 \\
\hline Deviation & $9.96 \mathrm{e}-61$ & $1.97 \mathrm{e}-58$ & $9.96 \mathrm{e}-61$ & $1.97 \mathrm{e}-58$ & 0 & 0 & 0 \\
\hline
\end{tabular}

\begin{tabular}{|r|c|c|c|c|c|c|c|c|}
\hline state & $\mathbf{c s}_{B, s}$ & $\mathbf{c s}_{\text {sens,s }}$ & $\mathbf{p s}_{B}$ & $\mathbf{p s}_{a g g}$ & $\mathbf{p s}_{\text {anx }}$ & $\mathbf{e s}_{\text {anx }}$ & $\mathbf{e s}_{a g g}$ & $\mathbf{e s c}_{B}$ \\
\hline Maxima & & & & & & & & \\
\hline Time point & 196 & 196 & 196 & 196 & 196 & 196 & 196 & 196 \\
\hline Value & 0.189974 & 0.097537 & 0.688646 & 0.848264 & 0.848264 & 0.848264 & 0.848264 & 0.646615 \\
\hline Aggimpact & 0.189974 & 0.097537 & 0.688646 & 0.848264 & 0.848264 & 0.848264 & 0.848264 & 0.646615 \\
\hline Deviation & 0 & 0 & 0 & 0 & 0 & 0 & 0 & 0 \\
\hline Minima & & & & & & & & \\
\hline Time point & 406 & 406 & 406 & 406 & 406 & 406 & 406 & 406 \\
\hline Value & $2.77 \mathrm{e}-37$ & 0.087503 & 0.627566 & 0.796629 & 0.796629 & 0.739934 & 0.739934 & 0.627566 \\
\hline Aggimpact & 0 & 0.087503 & 0.627566 & 0.796629 & 0.796629 & 0.739934 & 0.739934 & 0.627566 \\
\hline Deviation & $2.77 \mathrm{e}-37$ & 0 & 0 & 0 & 0 & 0 & 0 & 0 \\
\hline
\end{tabular}

\section{Discussion}

In this paper a computational model of the internal processes of persons suffering from Borderline Personality Disorder was presented. The model was built as a temporalcausal network model according to the Network-Oriented Modelling approach presented in (Treur, 2016), incorporating the characteristics described by neuropsychological findings from the literature. The Borderline Personality Disorder is a rather complex disorder, difficult to address. Such a computational model for the Borderline Personality Disorder is new, as far as the authors know.

Characteristics such as identity diffusion, the absence of a distancing mechanism, impulsivity, reduced emotion control and poor monitoring, low empathic capacity were incorporated in the model by setting specific values to the parameters concerning the underlying mechanisms, such as connection weights and speed factors. The model was verified by mathematical analysis, with satisfying results.

The model can be the basis for a virtual patient model application and used by therapists to perform what-if simulations to get insight in the borderline phenomenon. It may also be extended by therapies to simulate the effect of them. These can be next steps for future research. 


\section{References}

1. American Psychiatric Association: Diagnostic and Statistical Manual of Mental Disorders. 5th ed. Arlington, VA: American Psychiatric Association (2013)

2. Baker, A.E.Z., Lane, A.E., Angley, M.T., Young, R.L.: The relationship between sensory processing patterns and behavioural responsiveness in autistic disorder: a pilot study. J. Autism Dev. Disord. 38, 867-87 (2008)

3. Brand, B.L., and Lanius, R.A: Chronic complex dissociative disorders and borderline personality disorder: disorders of emotion dysregulation? Borderline Personality Disorder and Emotion Dysregulation 1, 13 (2014).

4. Brass, M., and Spengler, S.: The inhibition of imitative behaviour and attribution of mental states, in Social Cognition: Development, Neuroscience, and Autism. Ed. by T. Striano, V. Reid. Wiley-Blackwell, pp 52-66 (2009).

5. Corden, B., Chilvers, R., Skuse, D.: Avoidance of emotionally arousing stimuli predicts social-perceptual impairment in Asperger's syndrome. Neuropsychologia 46, 137-147 (2008)

6. Dammann, G., et al.: The Self-Image in Borderline Personality Disorder: An in-Depth Qualitative Research Study. Journal of Personality Disorders 25(4), 517-527 (2011).

7. De Meulemeester, C., Lowyck, B., Vermote, R., Verhaest, Y., Luyten, P.: Mentalizing and interpersonal problems in borderline personality disorder: The mediating role of identity diffusion. Psychiatry Research 258, 141-144 (2017).

8. De Vignemont, F., Singer, T.: The empathic brain: how, when and why? Trends Cogn. Sci. 10, 437-443 (2006).

9. Ellison, W.D., Rosenstein, L., Chelminskil, I., Dalrymple, K., Zimmerman, M.: The clinical significance of single features of borderline personality disorder; anger, affective instability, impulsivity, and chronic emptiness in psychiatric outpatients. J Pers Disord. 30( 2), 261-270 (2016).

10. Grèzes, J., de Gelder, B.: Social perception: understanding other people's intentions and emotions through their actions, in Social Cognition: Development, Neuroscience, and $\mathrm{Au}-$ tism. Ed. by T. Striano, V. Reid. Wiley-Blackwell, pp. 67-78 (2009).

11. Grèzes, J., Wicker, B., Berthoz, S., de Gelder, B.:A failure to grasp the affective meaning of actions in autism spectrum disorder subjects. Neuropsychologica 47, 1816-1825 (2009).

12. Haas, B.W., and Miller, J.D.: Borderline personality traits and brain activity during emotional perspective taking. Personality Disorders: Theory, Research, and Treatment 6(4), 315320 (2015).

13. Homan, P., et al.: Aberrant link between empathy and social attribution style in borderline personality disorder. Journal of Psychiatric Research 94, 163 - 171 (2017)

14. Iacoboni, M.: Mirroring People: The New Science of How We Connect with Others. Farrar, Straus \& Giroux. (2008).

15. Iacoboni, M., Dapretto M.: The mirror neuron system and the consequences of its dysfunction. Nat. Rev. Neurosci. 7, 942-951 (2006)

16. Kim, J.: Philosophy of Mind. Westview Press, 1996.

17. King-Casas, B, Sharp, C., Lomax-Bream, L., Lohrenz, T., Fonagy P., Montague, P.R.: The rupture and repair of cooperation in borderline personality disorder. Science. (2008).

18. Koenigsberg, H.W., Fan, J., Ochsner, K., Liu, X., Guise, K.G., Pizzarello, S., Dorantes, C., Guerreri, S., ecuta, L., Goodman, M., New, A., Siever, L.J.: Neural correlates of the use of psychological distancing to regulate responses to negative social cues: a study of patients with borderline personality disorder. Biol Psychiatry 66 (9), 854-863 (2009).

19. Kuipers, B.J.: Commonsense reasoning about causality: Deriving behavior from structure, Artificial Intelligence 24, 169-203 (1984). 
20. Kuipers, B.J., and Kassirer, J.P.: How to discover a knowledge representation for causal reasoning by studying an expert physician. In: Proc. of the 8th International Joint Conference on Artificial Intelligence, IJCAI'83, Karlsruhe. William Kaufman, Los Altos, CA (1983).

21. Lis, S., and Bohus, M.: Social Interaction in Borderline Personality Disorder. Curr Psychiatry Rep 15: 338. https://doi.org/10.1007/s11920-012-0338-z (2013).

22. Hall, M.A., and Riedford, K.M.: Borderline Personality Disorder: Diagnosis and Common Comorbidities. The Journal for Nurse Practitioners 13(9), e455-e456 (2017).

23. NIH Website

https://www.nimh.nih.gov/health/topics/borderline-personality-disorder/index.shtml https://www.nimh.nih.gov/news/science-news/2008/emotion-regulating-circuit-weakenedin-borderline-personality-disorder.shtml

24. Neumann, D., Spezio, M.L., Piven, J., Adolphs, R.: Looking you in the mouth: abnormal gaze in autism resulting from impaired top-down modulation of visual attention. Soc. Cognit. Affect.Neurosci. 1, 194-202 (2006).

25. Pearl, J.: Causality. Cambridge University Press (2000).

26. Singer, T., Leiberg, S.: Sharing the emotions of others: the neural bases of empathy. The Cognitive Neurosciences, 4th ed., ed. by M.S. Gazzaniga MIT Press, pp. 973-986 (2009).

27. Soloff, P., et al.: "Structural Brain Abnormalities in Borderline Personality Disorder: A Voxel-Based Morphometry Study." Psychiatry Research 164.3, 223-236 (2008) (2017).

28. Spezio, M.L., Adolphs, R., Hurley, R.S.E., Piven, J.: Analysis of face gaze in autism using 'Bubbles'. Neuropsychologia 45, 144-151 (2007).

29. Treur, J.: Network-Oriented Modeling: Addressing Complexity of Cognitive, Affective and Social Interactions. Springer (2016). 